\title{
Spatio-temporal probing of apparent rotational movement
}

\author{
CLIVE ROBINS and ROGER N. SHEPARD \\ Stanford University, Stanford, California 94305
}

\begin{abstract}
A visual bar alternately presented in vertical and horizontal orientations appeared to rotate $90^{\circ}$ through one of two pairs of opposite quadrants. Subjects judged whether a probe dot, interjected at some delay and angular deviation from the vertical bar, appeared before or after the bar "passed through" the corresponding angular orientation. When the motion was perceived in the probed quadrant, percent "before" responses dropped abruptly from near $100 \%$ to near $0 \%$ as delay increased, and the drop occurred at longer delays for probes at larger angular deviations. Under physically identical conditions, when the motion was perceived in an unprobed quadrant, percent "before" responses varied much less with delay and insignificantly with angular position. The accuracy of the judgments in the first case suggests the internal generation of an ordered sequence of intermediate representations during each apparent rotation.
\end{abstract}

Recent debate in cognitive psychology has concerned the nature of internal representations of real or imagined objects and of transformations on them. Many writers have favored discrete, symbolic, or propositional representations for visual as well as linguistic events, while others have presented evidence for more holistic or analogical representations of visual events [see Pylyshyn (1973) and Shepard and Podgorny (in press) for contrasting reviews of the alternative approaches]. Taking the second type of approach, Shepard and Metzler (1971) demonstrated that the time to determine whether two perspective line drawings portrayed the same threedimensional shape increased in a remarkably linear way with the angular difference between the portrayed orientations of the objects in threedimensional space. They suggested that a mental representation of one object was mentally "rotated" at a constant rate until it was brought into congruence with the other. Later studies of the transformation of mental images, including size transformations, reflections, and more complex structural transformations, have also indicated that imagined transformations of spatial objects bear important similarities to perceptions of corresponding actual transformations of externally presented objects [see Cooper and Shepard (in press) for a recent review].

One promising line of attack on the representation problem has employed apparent movement, a phenomenon intermediate between merely imagined and veridically perceived transformations. Taking as their starting point Korte's (1915) third law of

This work was supported by National Science Foundation Grant BNS-75-02806 to the second author. The authors are also indebted to Merrill Carlsmith for helpful suggestions concerning the statistical analyses. apparent movement that "an increase in the spatial separation between two stimuli must be accompanied by an increase in their temporal separation for an optimal appearance of movement to occur," Attneave and Block (1973) demonstrated that the critical spatial separation for Korte's law is the subjectively perceived distance in three-dimensional space and not the objective distance on the two-dimensional retina. More recently Shepard and Judd (1976) alternately presented Shepard and Metzler's (1971) pictures of three-dimensional objects differing in angular orientation. For appropriate time constants, they obtained a perceptual illusion of apparent rigid rotation even for views differing by as much as $180^{\circ}$. Moreover, Shepard and Judd found that the minimum onset-onset time necessary for the appearance of coherent rigid rotation increased linearly with angular difference and with the same slope for rotations both in the picture-plane and in depth. Despite marked quantitative differences in absolute overall level, the similarity in relative internal pattern between these findings and those of Shepard and Metzler (1971) suggests a possible commonality between purely imagined transformations and the perceptually experienced illusion of apparent movement. In any case, the more direct dependence of the phenomenon on distal relations in the external threedimensional world than on proximal relations in the two-dimensional retinal image in both the experiments of Attneave and Block and of Shepard and Judd implicates relatively sophisticated internally constructed representations not readily explained in terms of two-dimensionally organized sensory mechanisms.

The present study investigates another respect in which the experience of apparent movement may partake of some of the functional richness of the 
perception of real movement. We ask subjects to judge whether a probe dot interjected at different temporal and spatial positions between the two stimuli appears before or after the apparently rotating image passes that point in space. If such judgments are made as a systematic monotonic function of the spatio-temporal position of the probe, this would constitute evidence for an analog nature both of the representation of the object and of its motion.

Possibly, of course, what goes on internally during such apparent movement has little or nothing in common with what goes on during the perception of a corresponding actual movement. As was originally suggested by Wertheimer (1912), the illusion that such a movement has occurred may represent a kind of retrospective neural "short circuiting" that follows the presentation of both stimuli, rather than any internal construction of a sequence of representations of intermediate orientations between the two stimuli. In this case, subjects might still be able to make the required judgments of "before" or "after" by a deductive inference based on the relative spatial position of the probe and its relative temporal position within the interstimulus interval. However, subjects should then perform similarly in a control condition in which, although the stimulus sequence is objectively identical, the path of apparent motion of the stimulus does not pass through the position of the probe. If, on the other hand, perceived rotation is rich enough to base the before/after judgment on a visual comparison of the image with the probe, while pure temporal estimation is relatively impoverished, then the judgments under the control condition should be closer to chance than those under the experimental condition.

To achieve these two physically equivalent conditions, we presented subjects with an inherently ambiguous alternation of a vertical and a horizontal bar with coincident centers. The motion was then seen either to pass back and forth through the northeast and southwest quadrants or through the northwest and southeast quadrants. The experimental and control conditions, respectively, were achieved by presenting the probe in a quadrant through which the movement currently was or was not being perceived by that subject.

\section{METHOD}

\section{Subjects}

Twelve students and staff members at Stanford University served as subjects in two sessions of $1 \mathrm{~h}$ each.

\footnotetext{
Apparatus and Stimuli

Subjects viewed the repeating sequence of stimuli in an Iconix 6192 four-channel tachistoscope. As illustrated in Figure 1A, a total cycle time of $760 \mathrm{msec}$ was composed of the following displays, in this order: (1) a vertical white bar for $200 \mathrm{msec},(2)$ an empty black field for a variable preprobe interval (or $\mathrm{ISI}_{1}$ ), called
}

the probe delay, of between 30 to $130 \mathrm{msec}$ duration (in steps of $20 \mathrm{msec}$ ), (3) a small yellow probe dot in one of the upper quadrants for $20 \mathrm{msec}$, (4) a postprobe black interval (or ISI $_{2}$ ) for between 30 to $130 \mathrm{msec}$ so that the preprobe, probe, and postprobe intervals always totaled $180 \mathrm{msec}$, (5) a horizontal white bar for $200 \mathrm{msec}$, and (6) another black interval for $180 \mathrm{msec}$ before the whole cycle was repeated, starting with (1), above. The white bars and yellow probe dot were presented against the same black background used for the blank intervals. The vertical and horizontal bars were positioned such that their centers, marked by small black dots, coincided at the center of the display. Each bar measured $8.9 \times 1.3 \mathrm{~cm}$, which, at the approximately $90-\mathrm{cm}$ viewing distance, produced a visual angle of $5^{\circ} 40^{\prime}$. The $0.6-\mathrm{cm}$ yellow probe dot subtended a visual angle of $25^{\prime}$, and always appeared in one of the two upper quadrants at an angular position of $30^{\circ}, 40^{\circ}, 50^{\circ}$, or $60^{\circ}$ from vertical along a circular arc at $3.2 \mathrm{~cm}$ from the "pivotal" center of the display (as illustrated in Figure 1C for the right-hand quadrant).

\section{Procedure}

The subjects were read the following instructions while they viewed binocularly the described sequence of stimuli: "This experiment is concerned with apparent movement. I will display a vertical bar and a horizontal bar, alternating in rapid succession. This may give the appearance of a movement through space of a bar, rocking back and forth. Do you see that? Through which quadrants does the bar seem to pass? Now a yellow dot should appear at some point in the bar's path, during the downswing of the top end. Your task is to say whether the dot appears before or after the bar passes the dot's place of appearance. If at any time the direction of rotation should change for you, please let me know and the dot position will be changed accordingly. You may find the judgment rather difficult and you should take as long as you need to feel really certain about it. Your response will not be timed. Please do not guess unless and until you have really tried to decide and cannot. Do not expect there always to be an equal number of 'before' and 'after' responses for any given dot position, indeed do not make any assumptions about their relative frequencies but judge each trial independently. After each block of 12 trials you may rest while I change the position of the dot. There will be two types of blocks of trials. In one case, the dot will appear in a quadrant through which the bar seems to pass, as you have just seen; in the other case, the dot will be in a quadrant through which the bar is not passinglike this. In this case you are to make the analogous judgment, that is, whether the probe appears before or after the bar would pass that point if it were rotating in the opposite direction, or if the probe were in the symmetrically opposite position."

We did not instruct subjects to fixate their eyes in any particular way. (The small black dot at the center of each bar was intended only as a point of pivot for the apparent rotation.) Every subject reported seeing apparent rotation when queried, though the rotation varied over subjects, almost always being seen as confined either to quadrants 1 and 3 or to quadrants 2 and 4 , as shown in Figure 1B. Reported reversals in the quadrants of apparent movement occurred fairly infrequently, unexpectedly, and seemingly under little or no voluntary control.

Once we determined the quadrants through which rotation was being perceived by a given subject, we arranged to introduce the probe dot into the sequence in that one of the upper two quadrants appropriate to the experimental or control condition. If the quadrants of perceived rotation changed during a block of trials, the dot was shifted to the other side accordingly, such that the two main conditions were defined by a rotational direction/ dot-location combination. For each of these conditions there were four blocks of 12 trials, one block at each probe position of $30^{\circ}$, $40^{\circ}, 50^{\circ}$, and $60^{\circ}$. In a given block, each of the six permissible probe delays occurred twice, all trials being randomized independently for each block.

Each of the 12 subjects was run in two 1-h sessions of four 


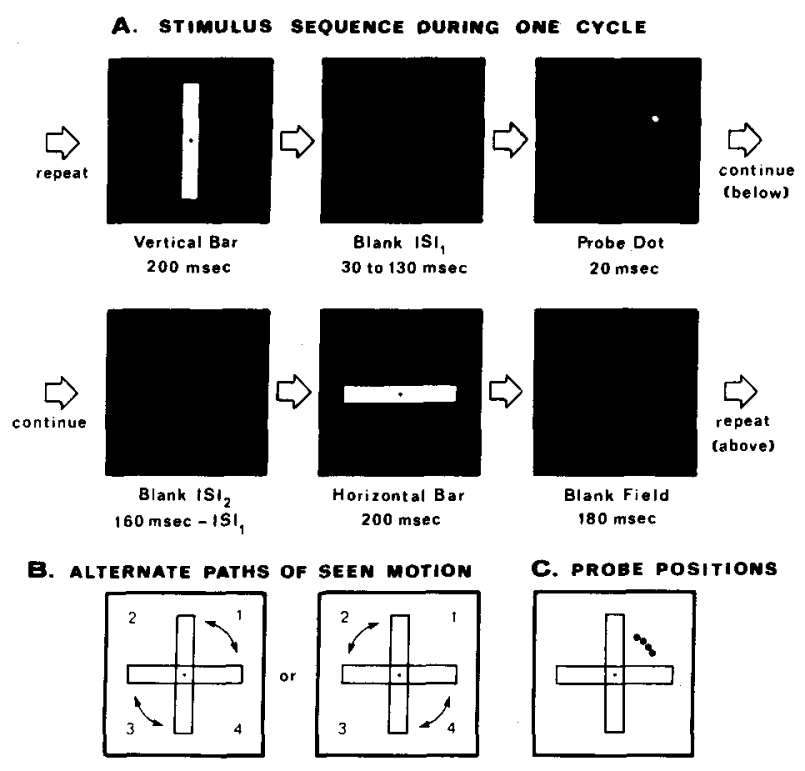

Figure 1. The sequence of visual fields presented during a single cycle (A), the two alternative paths through which the bar was virtually always seen to move (B), and the four angular positions in which the yellow probe dot might be presented-illustrated for one of the two upper quadrants only (C).

blocks, the sequence of blocks in any session being determined by a randomization within subjects with the restriction that each of the eight subconditions be balanced to occur three times in each of the four order positions over the whole set of 24 sessions. Since the same range of probe delays was employed for all dot positions, the expected ratio of "before" to "after" responses is not equal to .5 and varied systematically with probe position. By our instructions to treat trials independently, we attempted to discourage possible guessing strategies that might utilize this dependence of expected ratio on probe position.

At the beginning of each session, we gave subjects six practice trials in each of the two main conditions, in a randomized order of conditions and trials within conditions. Following those, the four blocks were run in the same manner, with responses recorded. No feedback was given either during practice or data collection. We noted any trials on which the perceived motion switched into the other two quadrants. Subjects were questioned informally after each session about their experience in the task generally, and specifically about any such switches, and about the nature and relative difficulty of the judgments in each of the two main conditions.

\section{RESULTS AND DISCUSSION}

At the top of Figure 2, the group data for the number of "before" responses are plotted as a function of probe delay for each of the four probe positions of $30^{\circ}, 40^{\circ}, 50^{\circ}$, and $60^{\circ}$. These four curves are plotted separately for the experimental condition, in which the probe was presented in the quadrant where the motion was being seen (Panel A) and for the control condition, in which the probe was presented in the opposite quadrant (Panel B). Of central interest are the different ways in which the frequency of "before" responses depends, in the two conditions, on probe delay and probe position.

\section{Effect of Probe Delay}

As is clear in the figure, the functional dependence of frequency of "before" responding on the delay of the probe was uniformly much stronger in the experimental than in the control condition; all four curves for the experimental condition (A) exhibit an abrupt, monotonic S-shaped decline from the maximum possible to zero "before" responses as probe delay increased, while all four curves for the control condition (B) are relatively much flatter and, indeed, never approach $100 \%$ or $0 \%$ responses.

For purposes of statistical analysis of the difference in dependence on delay in the two conditions, we computed the slope of the linear function of delay best fitting each subject's data after combining over all probe positions. For the experimental condition, all 12 of the subjects had negative slopes and the $95 \%$ confidence interval on the mean of these slopes was -.093 to -.077 . For the control condition, 10 of the 12 subjects had negative slopes and the $95 \%$ confidence interval was -.048 to -.012 . The slopes for the experimental condition were very significantly different from zero, $\mathrm{t}=24.58, \mathrm{p}<.001$, and those for the control condition, though shallower, were also significantly different from zero, $t=3.56$, $p<.01$. The difference in slopes between the two conditions, however, was also significant with $\mathrm{t}=2.57, \mathrm{p}<.05$.

\section{Effect of Probe Position}

For the experimental condition (Figure 2A), the four individual curves appear, furthermore, to be systematically displaced farther and farther to the right as the angle of the probe's position is increased from $30^{\circ}$ to $60^{\circ}$. But such a systematic shift with probe position is not present in the four curves for the control condition (except for what might be interpreted as a rightward displacement of the $60^{\circ}$ curve).

For purposes of statistical analysis this time, the number of "before" responses as a function of position was determined for each subject by summing over all probe delays. Again, slopes of these functions were computed for each subject under each condition. Of the 12 subjects, 11 had positive slopes in the experimental condition (and the remaining slope was zero), but only 8 of the 12 subjects had positive slopes in the control condition. Ninety-five percent confidence intervals on the mean of these slopes were .009 to .021 for the experimental condition and -.005 to .017 for the control condition. The slopes for the experimental condition were significantly different from zero, $\mathrm{t}=5.21, \mathrm{p}<.001$, whereas the slopes for the control condition did not differ significantly from zero, $t=1.16, p>.20$. Furthermore, there was a significant difference between the slopes for the two conditions, $t=3.84$, $\mathrm{p}<.01$. 

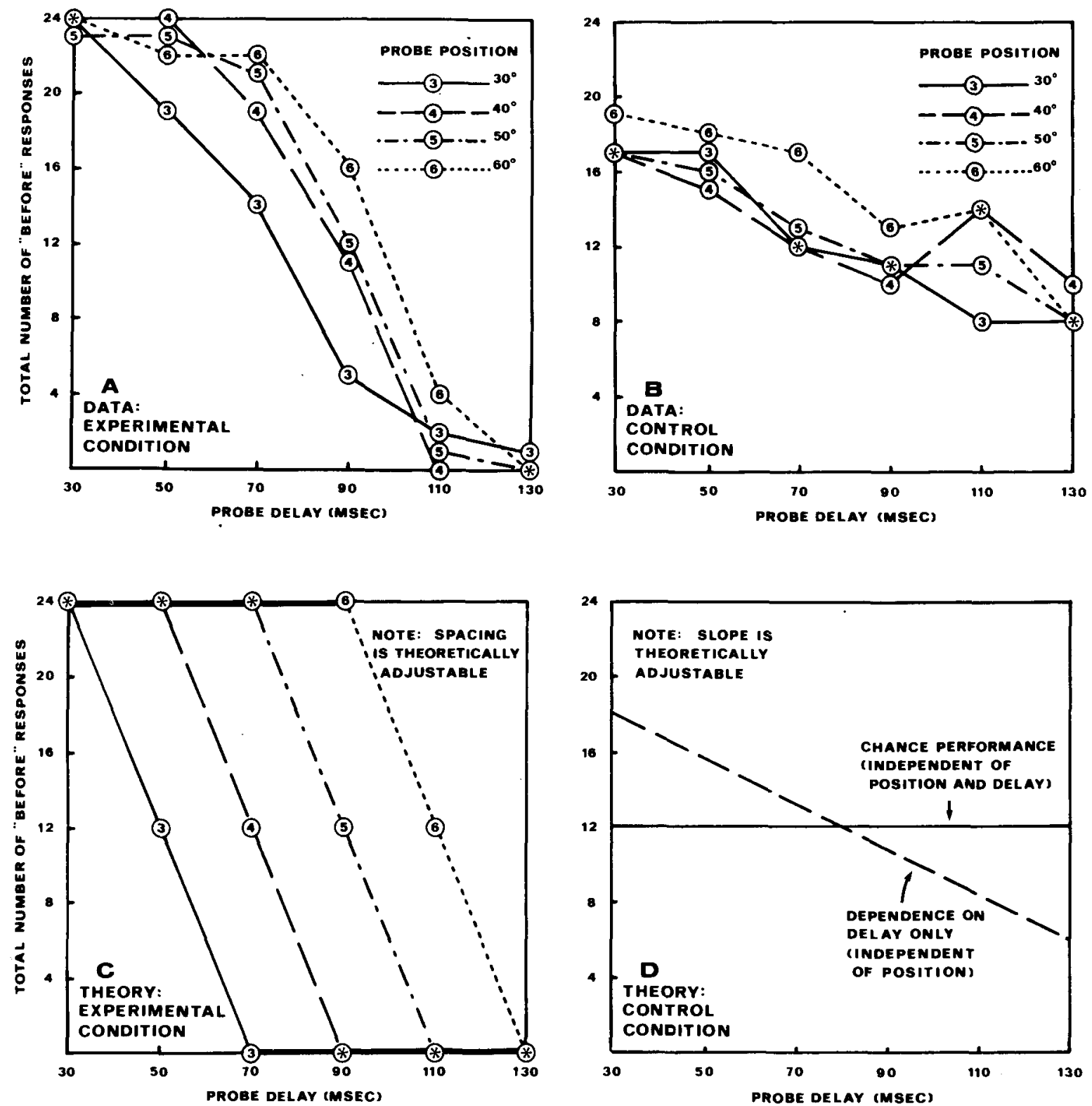

Figure 2. Curves, for each of the four probe positions, showing total number of "before" responses as a function of probe delay-as empirically obtained for the experimental condition in which the motion was perceived in the probed quadrant (A) and for the control condition in which the motion was perceived in the other quadrant (B), and as theoretically derived from alternative limiting assumptions $(C$ and $D)$.

These results are in agreement with the idea that subjects based their judgments in the experimental condition on a comparison between the probe and an internal representation of a bar rotating back and forth. Not only was there a virtually complete shift from consistent "before" to consistent "after" responding as the delay of the probe was increased by no more than about $40 \mathrm{msec}$, but the delay at which this shift occurred increased systematically with the angular distance of the probe along the path of the experienced motion. The results for the control condition, in which the stimulus sequences were physically identical but the experienced movement did not pass through the quadrant containing the probe, were in marked contrast. There was no abrupt shift from "before" to "after" responding with increasing delay, and the much smaller and more gradual shift that did occur was not related in a consistent monotonic way to the angular position of the probe.

\section{Comparison with Limiting Theoretical Cases}

In the absence of a detailed theory of the internal processes going on in subjects during apparent 
movement, it is not possible to make precise predictions about the actual probabilities of "before" and "after" responses at particular combinations of position and delay of the probe. Nevertheless, instructive comparisons can be made with theoretical predictions from two limiting extreme sorts of assumptions. One is that subjects respond "before" or "after" entirely randomly with equal probability, regardless of probe delay. In this case, the curves for all four probe positions should fall along the horizontal line labeled "chance performance" in Figure 2D. The other extreme sort of assumption is that subjects respond on the basis of a direct comparison between the probe and an internal representation of a bar actually rotating between vertical and horizontal positions. In this case, subjects should always respond "after" or "before," respectively, in accordance with whether the internal representation of the rotating bar has or has not yet passed the position of the probe at the moment of its presentation and should give both responses with probability .5 when the probe coincides with the momentarily represented position of the bar. The predicted curves then would take on the shapes illustrated in Figure 2C.

The lateral placements of these curves, however, would depend on the particular time course that we assume for the internally represented rotation. The curves displayed in Panel $\mathrm{C}$ are based on the quite specific assumption that this rotation proceeds at a constant rate beginning with the vertical orientation precisely at the perceptual offset of the vertical bar and ending with the horizontal orientation precisely at the perceptual onset of the horizontal bar. Although we shall argue below that this assumption is oversimplified and may need modification both on grounds of plausibility and in order to fit the data, the assumption provides a simple and well-defined limiting case for purposes of initial comparison.

Clearly, the data for the experimental condition are much closer to the limiting case in which the subjects are assumed to base their judgments on comparison with an internal representation of a rotating bar, while the data for the control condition are much closer to the limiting case in which the subjects are assumed to make their judgments completely at random. However, the obtained curves also suggest that the subjects were not always operating at the extreme limit represented by one of these theoretically pure cases.

In particular, if, in the control condition, subjects were able to make some crude estimate of the purely temporal position of the probe within the $180-\mathrm{msec}$ interstimulus interval, this could explain the consistent, though much weaker, decline in percent "before" judgments with probe delay shown theoretically by the dashed line in Figure $2 \mathrm{D}$ and empirically by the four curves for the control condition in Figure 2B. The notion that the judgments were thus based primarily on a purely temporal comparison in this condition, rather than on a spatiotemporal comparison with an internally represented moving bar as assumed for the experimental condition, would explain the lack of systematic dependence of the four curves in Figure $2 B$ on the spatial position of the probe.

Despite these indications, every subject, when questioned, claimed to have attempted to use the apparent movement as a basis for judgment even (though with much greater difficulty) in the control condition. Most commonly, they described trying simultaneously to focus attention on the timing of the probe and the corresponding position in the opposite quadrant in relation to the moving bar. Indeed, the moderate slope of the functions plotted for the group of subjects in the control condition (Figure 2B) heavily reflects the data of 2 of the 12 subjects who reported that, with practice, they were able to develop strategies using the apparent movement in this way that were nearly as effective in the control as in the experimental condition. (These two subjects, incidentally, were the two who had had the most previous experience in making judgments of this kind in our laboratory.)

In fact, judgments of purely temporal order of events within a repeating sequence are remarkably difficult-much more so, apparently, than for two isolated events (Hill \& Bliss, 1968; Sternberg \& Knoll, 1973, p. 674; Swisher \& Hirsh, 1972). In our own pilot work in the visual modality, we substituted homogeneous red and blue fields for the vertical and horizontal bars so that the temporal information was preserved but the spatio-temporal information contained in the motion perceived between the bars was entirely eliminated. The resulting experience was one of the whole background alternately flashing blue and red and, superimposed on this, a small dot flashing yellow with, however, a maddening absence of any determinable temporal relationship between the flashing dot and the flashing background. And, in summarizing the findings of several earlier studies using other modalities, Sternberg and Knoll (p. 674) concluded that "even when onsets of successive stimuli are separated by $100 \mathrm{msec}$ or more in recycling presentation, accuracy is far from perfect." The relatively gradual decline of the curves in Figure 2B over a range of probe delays from 30 to $130 \mathrm{msec}$ appears consistent with this conclusion.

Even though we have some reservations about the specific assumptions underlying the theoretical curves exhibited in Figure $2 \mathrm{C}$, we attempted a statistical analysis of the degree to which the data from the two conditions departed from these curves. We defined "errors" as responses that were opposite from what would have been correct responses if a bar had actually been rotating at a constant rate between the offset of the vertical and onset of the horizontal 
bar-that is, as responses of "before" or "after" opposite to what is indicated by the relevant solid curve in Figure $2 \mathrm{C}$. By this definition, every subject made more errors in the control condition than in the experimental condition. For probe positions of $30^{\circ}, 40^{\circ}, 50^{\circ}$, and $60^{\circ}$, respectively, the mean number of errors (out of 10 possible) were 1.83, $0.92,0.42$, and 0.92 in the experimental condition, and $3.92,4.17,3.58$, and 3.08 in the control condition. These differences between the experimental and control condition were all statistically significant; for the same four probe positions, $\mathrm{t}_{11}$ was $3.1,5.4,6.5$, and 4.4 , respectively, with $\mathrm{p}<.01$ according to the two-tailed test in every case. The objective results are thus consonant with the subjects' reports that the control condition was always more taxing than the experimental condition, and with the experimenter's informal impression that the judgment latencies, though not recorded, were generally about two to three times longer for the control than for the experimental condition.

\section{Explanations for Departures from \\ Theoretical Curves}

Of course, we don't believe the specific assumptions underlying the theoretical curves in Figure $2 \mathrm{C}$ to be quite correct. In the experimental condition, any fuzziness or variability in the internal representation of the rotating bar would be expected to entail some rounding off of the curves-perhaps as found in Figure 2A. (And this would be expected in the perception of real movement as well as in this illusion of apparent movement.) Moreover, there is reason to doubt the assumption that internal rotation commences at the offset of one bar and ends at the onset of the next bar. In accordance with Korte's "fourth law," apparent movement has been shown to depend more on onset-onset time than on the interstimulus interval (e.g., see Kahneman, 1967) and, even in the case of apparent rotation, is much the same when this interval is reduced to zero (Shepard \& Judd, 1976). In the present case, by assuming that the internal representation of the rotation begins quite a bit later and ends slightly earlier (and, possibly, accelerates and then decelerates), we can predict curves that differ from those in Figure $2 \mathrm{C}$ in being relatively shifted to the right and compressed together. In this way, the theoretical curves could be brought into a closer approximation to the empirical curves for the experimental condition (Panel A). However, we presently have little basis on which to argue for any particular starting or stopping times for the internally represented rotation (and, in view of the linear functions obtained by Shepard and Judd, we prefer the working assumption that the rate of the represented rotation, once started, is essentially constant). Furthermore, there are other factors that may contribute to the rightward shift and relative compression of the four curves in Figure $2 \mathrm{~A}$.

In particular, when we ourselves played the role of subject, it appeared to us that, when the probe was close to the vertical bar (i.e., at $30^{\circ}$ ), the strong afterimage of the bar "above" the probe induced a strong bias to report "before," while, when the probe was close to the horizontal bar (i.e., at $60^{\circ}$ ), the weaker afterimage of the probe above the bar induced a bias (though probably a weaker one) to report "after." Possibly, the biasing effect of such afterimages could be decreased by reducing the width and brightness contrast of the visual bar.

\section{Spontaneous Switches in Quadrant of Perceived Motion}

A striking aspect of apparent movement with such inherently ambiguous stimulus displays is the categorically distinct phenomenal appearances of the different ways of perceiving motion under identical stimulus conditions. Seven of the 12 subjects experienced spontaneous switches in the quadrants ofthe perceived motion during the experiment. Of the 31 such switches reported, 19 occurred in the control condition and 12 in the experimental-a nonsignificant difference. Eleven reversals of the 31 occurred on trials in which the probe and bar should, according to the special assumptions underlying the curves in Figure $2 C$, appear simultaneous. This is significantly greater than expected by chance $\left[\chi^{2}(1)=7.89\right.$, $\mathrm{p}<.01$, two-tailed]. Informal observation suggests that the latencies on these trials averaged much longer than on other trials. The reversals might therefore be a consequence of greater cognitive strain or simply of the greater time available for the occurrence of a reversal.

\section{CONCLUDING REMARKS}

No one experiment can resolve all of the issues concerning the internal representation of apparent movement. We believe, however, that the use of spatiotemporally localized probes during the course of a perceptually related internal transformation shows promise for clarifying the extent to which the internal representations of objects and their transformations are analogical in the sense of having a one-to-one relation to corresponding external objects and events. In the specific case of apparent movement, our results, together with those of Shepard and Judd (1976), appear to furnish some support for the idea that, when under identical stimulus conditions a bar is seen to be rocking back and forth either one way or the other way, it is not merely an illusion in which the subject has the retrospective impression that a motion of the one sort or 
the other has occurred. The precision of the subject's judgments of the relation of the bar to the probe during the course of the perceived motion suggests that a sequence of representations of the bar in successive intermediate orientations may at least schematically have been generated. (Our data do not, however, bear on the question of whether such an intermediate sequence is discrete or continuous.)

Of course, if such an intermediate sequence is internally generated, it may only be generated after the presentation of the second stimulus. Logically, this would have to be the case upon the first presentation of the second stimulus since, without knowing what that stimulus was to be, the subject could not know what intermediate sequence to generate. However even "direct" perception takes time, and so it is quite likely in our experiment, as Beck, Elsner, and Silverstein (1977) established in their very recent study of apparent movement, that the internal representation of the movement between two successively presented stimuli (like the internal representation of those stimuli themselves) follows the external events with a time lag comparable to the interstimulus interval.

Finally, our conclusions might seem to be inconsistent with Kolers' (1963) finding that the apparent movement of a line did not mask a spatiotemporally interposed real line in the way that the corresponding real movement of a similar line did. However, it may be that the internally generated sequence of intermediate represertations that we are tentatively postulating is at an abstract representational level too far removed from the peripheral mechanism to produce this kind of sensory masking. The priority of distal relations in three-dimensional space over proximal relations on the two-dimensional retina, noted at the outset, points in this direction. In our own experiment, sensory detectors corresponding to the physical superposition of the yellow dot on the white bar presumably would not themselves ever have been stimulated. It is not surprising, then, that several of our subjects reported that, when the dot flashed just as the bar appeared to pass through that position, the dot and bar themselves seemed strangely to elude or to jump around each other without yielding the unmistakable appearance of direct superposition. Nevertheless, the higher level representation of the close encounter between the flashing dot and the moving bar evidently sufficed to permit a remarkably refined temporal judgment.

\section{REFERENCES}

Attneave, F., \& Block, G. Apparent movement in tridimensional space. Perception \& Psychophysics, 1973, 13, 301-307.

Beck, J., Elsner, A., \& Silverstein, C. Position uncertainty and the perception of apparent movement. Perception \& Psychophysics, 1977, 21, 33-38.

COOPER, L. A., \& ShEPARD, R. N. Transformations on representations of objects in space. In E. C. Carterette \& M. P. Friedman (Eds.), Handbook of perception (Vol. VIII) Space and object perception. New York: Academic Press, in press.

Hill, J. W., \& Bliss, J. C. Perception of sequentially presented tactile point stimuli. Perception \& Psychophysics, 1968, 4, 289-295.

Kahneman, D. An onset-onset law for one case of apparent motion and metacontrast. Perception \& Psychophysics, 1967, 2. 577-584.

Kolers, P. A. Some differences between real and apparent visual movement. Vision Research, 1963, 3, 191-206.

KORTE, A. Kinematoskopische Untersuchungen. Zeitschrift für Psychologie, 1915, 72, 193-296.

Prlyshyn. Z. What the mind's eye tells the mind's brain: A critique of mental imagery. Psychological Bulletin. 1973. 80. 1-24.

ShePard, R. N., \& JUdd, S. A. Perceptual illusion of rotation of three-dimensional objects. Science, 1976, 191, 952-954.

Shepard, R. N., \& METzler, J. Mental rotation of threedimensional objects. Science, 1971, 171, 701-703.

Shepard, R. N., \& Podgorny, P. Cognitive processes that resemble perceptual processes. In W. K. Estes (Ed.), Handbook of learning and cognitive processes. Hillsdale, N.J: Erlbaum, in press.

Sternberg, S., \& KNoll, R. L. The perception of temporal order: Fundamental issues and a general model. In $S$. Kornblum (Ed.), Attention and performance IV. New York: Academic Press, 1973.

Swisher, L., \& Hirsh, I. Brain damage and the ordering of two temporally successive stimuli. Neuropsychologia, 1972, $10,137-152$.

Wertheimer, M. Experimentelle Studien über das Sehen von Bewegung. Zeitschrift für Psychologie, 1912, 61, 161-265.

(Received for publication March 11, 1977; accepted May 4, 1977.) 